\title{
Comment on: Zoonosis: an unusual case of chronic osteomyelitis
}

Singapore Med J 2019; 60(10): 553 https://doi.org/10.11622/smedj.2019117

Dear Sir,

We read the letter 'Zoonosis: an unusual case of chronic osteomyelitis' with great interest. ${ }^{(1)} \mathrm{Ng}$ et al mentioned that "this case report adds to the current limited understanding of Edwardsiella infections in humans and potentially represents the first observational evidence that Edwardsiella may persist within human tissues for a prolonged period of time" . $^{(1)}$ We would like to point out that in fact, Edwardsiella anguillarum infections are rarely reported in humans and are difficult to diagnose if the practitioner does not recognise them. Therefore, infection of the bone by this pathogen might be prolonged before a final diagnosis is derived. The authors' case report is also not the first. There are already some reports in the literature regarding chronic bone infection due to this pathogen.(2-4) Generally, patients who have bone infection due to this pathogen usually have underlying disease, especially immune-haematological problems. ${ }^{(2-4)}$

Yours sincerely,

Rujittika Mungmunpuntipantip ${ }^{1}$, Viroj Wiwanitkit ${ }^{2,3}$

${ }^{1} 26$ Medical Center, Bangkok, Thailand, ${ }^{2} J o s e p h$ Ayo Babalola University, Ikeji-Arakeji, Osun, Nigeria, ${ }^{3}$ Dr DY Patil University, Pune, India. rujittika@gmail.com

\section{References}

1. Ng QX, Seng C, Chan FZY, Yeo WS. Zoonosis: an unusual case of chronic osteomyelitis. Singapore Med J 2019; 60:379-81.

2. Janda JM, Abbott SL. Infections associated with the genus Edwardsiella: the role of Edwardsiella tarda in human disease. Clin Infect Dis 1993; 17:742-8.

3. Ruff WL, Scott-Cora J, Prout JD. Edwardsiella tarda associated with osteomyelitis. J Natl Med Assoc 1977; 69:871-2.

4. Rao KR, Shah J, Rajashekaraiah KR, et al. Edwardsiella tarda osteomyelitis in a patient with SC hemoglobinopathy. South Med J 1981; 74:288-92.

Editor's note: The authors, Ng et al, have declined to respond to the above letter. 